\title{
La disciplina violenta, y el desarrollo cognitivo y socioemocional en el infante de preescolar
}

\author{
DANIELA PAZOS POLO \\ MARÍA SÁNCHEZ TRUJILLO ${ }^{*}$ \\ Pontificia Universidad Católica del Perú - Perú \\ Recibido el 12-02-20; primera evaluación el 05-01-21; \\ segunda evaluación el 23-01-21; aceptado el 16-02-21
}

\section{RESUMEN}

El objetivo general del estudio fue describir la relación entre el estilo de disciplina ejercido por la docente del aula de 4 años de una institución educativa privada de Lima, y el desarrollo cognitivo y socioemocional de los estudiantes. En esta investigación, de naturaleza cualitativa descriptiva, se hizo uso de un cuaderno de campo y una entrevista semiestructurada dirigida a la docente. Entre los hallazgos más relevantes, resalta que las prácticas pedagógicas aplicadas en el aula vulneran y perjudican principalmente el desarrollo socioemocional de sus estudiantes, lo cual genera distracción y desinterés en clase. Ello, a su vez, podría estar asociado con el bajo desempeño académico de los estudiantes. Se concluyó que la docente necesita aplicar estrategias pedagógicas pertinentes y adecuadas según las necesidades del grupo de infantes.

Palabras clave: conducta violenta, desarrollo emocional, desarrollo cognitivo, estilos de disciplina, disciplina violenta.

\footnotetext{
* Educadora en formación de la especialidad de Inicial en la Pontificia Universidad Católica del Perú. Certificada por el IESP Emilia Barcia Boniffatti y por el Centro de Atención Temprana y Psicomotricidad Correo electrónico: daniela.pazos@pucp.edu.pe. https://orcid.org/0000-0003-1771-1266

** Doctora en Ciencias de la Educación. Magíster en Educación con dos menciones: Trastornos de la Comunicación y Gestión Educativa. Licenciada en Educación en la especialidad de Lengua y Literatura. Docente universitaria de cursos de Lenguaje, Redacción, Investigación Educativa y Metodología de Enseńanza. Correo electrónico: msanchezt@pucp.edu.pe. https://orcid.org/ 0000-0002-5228-4688
} 


\section{Violent discipline, and cognitive and socio-emotional development in the preschool infant}

\section{Abstract}

The general objective of the study was to describe the relationship between the style of discipline exercised by the teacher in the 4-year-old classroom of a private educational institution in Lima, and the cognitive and socio-emotional development of the students. In this research, of a qualitative descriptive nature, a field notebook and a semi-structured interview for the teacher were used. Among the most relevant findings, it stands out that the pedagogical practices applied in the classroom mainly violate and harm the socio-emotional development of their students, which generates distraction and disinterest in class. This, in turn, could be associated with the poor academic performance of students. It was concluded that the teacher needs to apply pertinent and adequate pedagogical strategies according to the needs of the group of infants.

Keywords: violent behaviour, emotional development, cognitive development, discipline styles, violent discipline

\section{Disciplina violenta e desenvolvimento cognitivo e socioemocional em crianças pré-escolares}

\section{Resumo}

O objetivo geral do estudo foi descrever a relaçáo entre o estilo de disciplina exercido pelo professor em uma sala de aula de 4 anos de uma instituição de ensino particular em Lima e o desenvolvimento cognitivo e socioemocional dos alunos. Nesta pesquisa, de natureza qualitativa descritiva, utilizou-se um caderno de campo e uma entrevista semiestruturada para o professor. Dentre os achados mais relevantes, destaca-se que as práticas pedagógicas aplicadas em sala de aula violam e prejudicam principalmente o desenvolvimento socioemocional de seus alunos, o que gera distração e desinteresse pela aula. Isso, por sua vez, pode estar associado ao baixo desempenho acadêmico dos alunos. Concluiu-se que o professor necessita aplicar estratégias pedagógicas pertinentes e adequadas de acordo com as necessidades do grupo de crianças.

Palavras-chave: comportamento violento, desenvolvimento emocional, desenvolvimento cognitivo, estilos de disciplina, disciplina violenta. 


\section{INTRODUCCIÓN}

Hace un tiempo, en el siglo XX, se implementaba, dentro de las aulas, una educación tradicional, en la cual el rol del educador consistía en impartir una autoridad opresiva y humillante frente a los educandos. En este espacio, se generaba un clima violento, en el que no se respetaban sus necesidades, intereses y demandas (Zovko y Dillon, 2018). Asimismo, desde ese enfoque, se consideraba, como objetivo único y prioritario, saturar de información las mentes del alumnado a través de una relación vertical y autoritaria entre docente y estudiante. La estimulación o potenciación de diferentes aspectos del desarrollo humano del infante como, por ejemplo, las áreas psicomotoras, cognitivas, del lenguaje y socioafectivas quedaban en segundo plano. Con el transcurso del tiempo, gracias a la evolución de las diversas teorías educativas, la concepción sobre la educación ha ido cambiando. Así, en la actualidad, se aboga por una educación democrática y basada en el desarrollo de competencias.

A pesar de lo anterior, un siglo después, en ciertas instituciones del país, aún se conserva aquella vaga y despreocupada corriente educativa (Prensky, citado en Larrańaga, 2012). Esta desigualdad y clima de convivencia contraproducente para el desarrollo del niño afecta su aprendizaje, así como su formación socioemocional, lo cual no siempre es tomado en cuenta en el sistema educativo actual (Pérez y Filella, 2019). En relación con ello, se ha comprobado que las competencias socioemocionales evidenciadas por los docentes en el ejercicio de sus funciones influyen en el éxito académico de los estudiantes (Llorent, Zych y Varo-Millán, 2020).

A partir de lo anterior, se buscó indagar acerca de los estilos de disciplina ejercidos por los docentes de educación inicial. Para ello, se eligió una institución ubicada en el distrito de Comas en el aula de 4 años, en la cual, a partir de experiencias previas en la labor de asistente por parte de una de las investigadoras en otra aula del centro educativo, se había observado un estilo docente supuestamente enmarcado en la disciplina violenta.

Al respecto, es pertinente mencionar al Minedu (2010, citado en Márquez, Díaz y Cazzato, 2007), que categoriza a la disciplina en obediente y consciente. La primera hace referencia a un trato vertical entre el docente y el alumno mediante el empleo de amenazas, condicionamientos y normas coactivas. Mientras, la disciplina consciente consiste en un trato horizontal y democrático en conjunto con normas establecidas por el docente y los estudiantes a través de las cuales aprenden a vivir en un clima de convivencia positivo. Evidentemente, desde un enfoque contemporáneo de la enseñanza, 
abogamos por este último, teniendo en cuenta la importancia de un entorno educativo favorable para el éxito académico de los estudiantes.

En correspondencia con lo señalado líneas arriba, se planteó, como objetivo general, describir la relación entre el estilo de disciplina ejercido por la docente, y el desarrollo cognitivo y socioemocional de los niños de 4 años de una institución educativa privada de Lima. Entre los objetivos específicos, se determinó, en primer lugar, identificar el estilo de disciplina ejercido por la docente; en segundo lugar, describir el desarrollo cognitivo de los estudiantes; y, en tercer lugar, describir el desarrollo socioemocional de los alumnos.

Cabe resaltar la importancia del presente estudio, pues ha permitido la investigación acerca de dos aspectos fundamentales de la práctica en el aula: el estilo de disciplina, y el desarrollo cognitivo y emocional de los estudiantes. Consideramos estos aspectos sumamente importantes, pues la manera en la que el docente conduce sus sesiones de aprendizaje representa un factor fundamental en el aprendizaje de los alumnos y en el desarrollo de sus competencias socioafectivas, todo lo cual les permitirá convertirse en ciudadanos responsables y valiosos para la sociedad (Guedeney y Dupong, 2020). Cabe indicar que estos aspectos no han sido estudiados con profundidad en el contexto de educación inicial, por lo cual el aporte del presente estudio resulta significativo.

\section{Marco teórico}

En relación con nuestro tema, como se ha mencionado anteriormente, no existen muchas investigaciones enmarcadas en el contexto de educación inicial. A continuación, se presentan algunos estudios que han servido de base para el presente.

En principio, en la investigación de Jiménez (2018), se buscó aplicar prácticas pedagógicas relacionadas con diferentes estrategias para corregir y modular el comportamiento de estudiantes a través del modelo educativo de disciplina positiva en alumnos de educación básica regular de instituciones educativas de Ecuador. Entre los resultados, destaca un mejoramiento del rendimiento académico por parte del alumnado. Dicho estudio se complementa con el efectuado por Silupu (2019) en una institución educativa privada de Lima, quien comprobó que los niños preescolares de 5 años reaccionan adecuadamente, en un $65 \%$, a un estilo de disciplina positiva.

Por otro lado, Chávez, Ramos y Velázquez (2017), en la investigación que realizaron con docentes de educación preescolar de México, constataron que las estrategias de disciplina positiva posibilitan mejores aprendizajes y, a su vez, contribuyen a la formación de la competencia socioemocional del alumnado. 
No obstante, pese a las diferentes estrategias que existen para promover este tipo de disciplina en los estudiantes, estudios como el realizado por Quispe (2018) han comprobado el deficiente manejo de estrategias didácticas para mejorar la disciplina de los alumnos. Así, investigaciones como la ejecutada por Bwire, Nkuba, Hermenau y Hecker (2020) permiten corroborar que un estilo de disciplina violenta aún prevalece en diversos lugares del mundo, lo cual perjudica el desarrollo académico y socioafectivo de los menores.

Por otra parte, Zamudio (2010) realizó una investigación orientada a determinar el impacto de un programa sobre formación de disciplina escolar en 504 profesores de educación primaria de diferentes escuelas adscritas al Subsistema Educativo Estatal de la Secretaría de Educación del Estado de México. Entre sus conclusiones, se plantea que, para conducir de manera viable la disciplina en las aulas, es preciso incorporar a los maestros programas diseñados en respuestas a sus necesidades. Asimismo, se afirma que la forma de atender a las necesidades disciplinarias en el aula exige desterrar creencias equivocadas basadas en la disciplina autoritaria como el método más eficaz de manejo de aula. A partir de tal punto, es preciso reforzar la idea del docente como ejemplo de inteligencia emocional, de modo que transmita actitudes positivas hacia sus estudiantes (Cabello, 2011).

Para comprender hacia qué sentido se orienta el trabajo de investigación, es fundamental conceptualizar ciertos términos claves.

\subsection{Estilos de disciplina}

El aula es el espacio en donde la disciplina funciona como una herramienta para conseguir determinados fines: socialización del alumnado, autonomía, rendimiento, autocontrol, etc. En cualquier caso, se trata de un concepto controvertido en el que confluyen distintas formas de entenderlo. Según Márquez et al. (2007), la disciplina puede ser concebida como medida correctora de conductas inadecuadas para desarrollar y controlar las conductas deseadas. Por otra parte, el Minedu (2010, citado en Córdova, 2013) categoriza a la disciplina en obediente y consciente, como ya se explicó anteriormente.

Ahora bien, es posible afirmar que, dependiendo de la manera como se lleve a cabo, la disciplina puede ser entendida como una acción positiva dentro del aula de clases, puesto que posibilita la formación actitudinal de los estudiantes en relación con un conjunto de normas preestablecidas, las cuales deben garantizar una convivencia pacífica y armoniosa entre todos los estudiantes. Por su parte, tal como señalan Aragão, Dos Santos y De Andrade (2017), la disciplina positiva concibe al alumnado como seres abiertos al 
diálogo, capaces de aprender de sus errores y de ser educados sobre la base del desarrollo y aceptación de su individualidad. Igualmente, según Nelsen (2015), la disciplina positiva no es equivalente a la permisividad; al contrario, implica una firmeza por parte del docente, pero sin llegar a ser autoritario. Desde tal perspectiva, su labor se orienta a construir una conciencia moral en sus estudiantes y a hacerlos responsables de sus propias acciones, reforzando las conductas positivas y minimizando las negativas.

En relación con el estilo docente, es importante mencionar también la clasificación realizada por Ellis y Hughes (2002), quienes señalan que existen cuatro tipos de estilo docente. En primer lugar, el autoritario: se caracteriza por priorizar un salón de clase ordenado y un alto nivel de desarrollo de tareas en desmedro del componente emocional y el clima de clase afectivo. En este tipo de estilo priman los castigos como medida correctora principal, pese a que se ha comprobado su ineficacia y, más bien, los perjuicios cognitivos y emocionales que pueden generar en los estudiantes (Soto, 2017). Cabe seńalar que las prácticas violentas en el aula de clase pueden llegar a ser normalizadas por los estudiantes, quienes incluso podrían reproducirlas posteriormente (Juárez y Márquez, 2010). Asimismo, como afirma Mazadiego (2005), este tipo de estilo constituye una forma de abuso y negligencia infantil. En segundo lugar, el estilo permisivo se caracteriza por la primacía del afecto del docente hacia sus estudiantes. En este estilo, se le otorga poca importancia a los límites y las normas. En tercer lugar, el estilo negligente, en el que los docentes no controlan adecuadamente a sus estudiantes ni tampoco les brindan afecto. Finalmente, en el estilo democrático-asertivo, el clima de aula es adecuado, y las normas de la clase son claras y precisas, lo que favorece las relaciones entre los estudiantes.

\subsection{Desarrollo cognitivo}

El desarrollo cognitivo es definido por Albornoz y Guzmán (2016) como un proceso por medio del cual el infante organiza mentalmente la información que recibe a través de los sistemas senso-perceptuales para la resolución de nuevas situaciones sobre la base de experiencias del pasado. Asimismo, Piaget (s/f, citado en Crain y Dunn, 2007) concibe el desarrollo del ser humano como un proceso de construcción constante, en el cual cada niño y niña, a través de sus propias prácticas, logra construir estructuras cognoscitivas cada vez más organizadas y completas, cuyo fin consiste en permitirle la adaptación al medio, lo que es concebido por el autor como el ajuste a nueva información del entorno a través de los procesos fundamentales de la cognición: la asimilación y la acomodación. Así, es preciso generar experiencias, desde los primeros ańos 
de vida, para mejorar las conexiones neuronales y, de esta manera, posibilitar un óptimo desarrollo de sus funciones cerebrales y cognitivas (Redolar, 2014). Como señala Santrock (2004), el desarrollo cognitivo involucra distintos aspectos relacionados con la estimulación sensorial, la exploración física y social, así el fortalecimiento de las operaciones cognitivas, tales como la atención, el razonamiento, la memoria, la metacognición y el aprendizaje (Best, 2002).

Ahora bien, de acuerdo con Pulido y Herrera (2017), el desarrollo cognitivo de los niños y adolescentes resulta influido por sus emociones. Si el estudiante no se encuentra motivado para el estudio o enfrenta estados emocionales negativos, ello le provocará no solo reacciones fisiológicas dañinas para su salud, sino también limitaciones en su aprendizaje y rendimiento académico. Incluso, como asevera Campos (2010), los altos niveles de estrés en el infante generan un efecto significativo en su aprendizaje al alterar la ejecución de sus operaciones cognitivas. Por tal motivo, resulta fundamental considerar el bienestar emocional de los estudiantes durante su proceso educativo, aspecto que se explicará en el siguiente apartado.

\subsection{Desarrollo socioemocional}

Los infantes se desarrollan e interactúan en espacios socioculturales distintos $y$, como consecuencia, también es distinto el proceso de desarrollo emocional (Sánchez, 2008). De manera análoga, desde los dos ańos en adelante, la vida emocional del infante está determinada por la influencia de la calidad y del tipo de interacción que pueda establecer con sus padres, familiares, docentes y pares. El diálogo entre adulto y nińo acerca de experiencias o eventos emocionales y las instrucciones del adulto cuidador sobre las expresiones emocionales más apropiadas para cada situación social ofrecen la posibilidad al infante de ir identificando algunas conductas emocionales de las que se va apropiando. De acuerdo con Bisquerra y Pérez (2007), el desarrollo emocional del niño implica el logro de la conciencia del propio estado emocional y la habilidad de discernir los propios sentimientos del de los demás, pero esto se logra solo a partir de la madurez alcanzada al haber formado estrategias de autocontrol adecuadas a partir de interacciones positivas exitosas. Evidentemente, en un clima de aula inadecuado, vinculado con interacciones negativas entre los discentes o con un estilo de disciplina inadecuado por parte del docente, el desarrollo emocional se verá perjudicado (Lafrancesco, 2003; Molina y Pérez, 2006; Espot, 2011). En tal caso, la imagen que proyecta el docente y sus propias habilidades de autorregulación emocional también influyen en la formación socioemocional de los estudiantes. 
Finalmente, Garaigordobil (2018), aboga por una educación emocional en la escuela, en la cual se incentive la formación de competencias socioemocionales fundamentales para mejorar el nivel de participación de los estudiantes en su comunidad y también para favorecer la convivencia. Al respecto, el tipo de disciplina que se imparta en clase favorecerá o, más bien, limitará el desarrollo de habilidades socioemocionales en los estudiantes. Así, si ejerce una disciplina positiva, los alumnos aprenderán ciertas actitudes importantes para su desarrollo personal y social, tales como manejar sus propias emociones, autorregularse, ser empáticos, entre otras. Cabe resaltar que el clima de clase en donde primen la seguridad, la protección y la afectividad es sumamente importante para el logro de un bienestar socioemocional por parte de los estudiantes. En esta línea, el empleo del juego resulta un elemento socializador relevante para el aprendizaje de los niños puesto que posibilita su aprendizaje de actitudes positivas en relación con situaciones de competitividad, además de interacciones sociales eficaces y del desarrollo de sus habilidades cognitivas, sobre la base de normas y negociaciones previamente determinadas. (Peralta, 2013).

\section{Metodología}

\subsection{Enfoque de investigación}

La investigación sigue un enfoque cualitativo y corresponde a un estudio de caso. Del mismo modo, el presente estudio es descriptivo, pues pretende lograr una comprensión profunda de la realidad a partir de las situaciones observadas.

\subsection{Informantes}

Los informantes son 6 niñas y 5 niños de 4 años, y una docente del nivel inicial de 35 años, pertenecientes a una escuela de gestión privada ubicada en el distrito 7 de Comas. Esta institución se caracteriza por ofrecer una formación personalizada e integral, además de declarar, entre sus principios rectores, la necesidad de trabajar en conjunto con la familia, formar a los estudiantes como futuros ciudadanos, y ofrecerles una educación religiosa basada en el catolicismo.

\subsection{Categorías de estudio}

Para el desarrollo del estudio, se consideraron tres categorías apriorísticas: estilo de disciplina, desarrollo cognitivo y desarrollo socioemocional de los infantes. En la tabla 1, se presentan las subcategorías consideradas. 
Tabla 1. Categorías y subcategorías de estudio

\begin{tabular}{ll}
\hline \multicolumn{1}{c}{ Categoría } & \multicolumn{1}{c}{ Subcategorías } \\
\hline Estilo de disciplina & $\rightarrow$ Obediente \\
& $\rightarrow$ Consciente \\
Desarrollo cognitivo & $\rightarrow$ Comprensión de la realidad física y social \\
& $\rightarrow$ Estimulación sensorial \\
& $\rightarrow$ Exploración física y social con su entorno \\
& $\rightarrow$ Desarrollo de los procesos cognitivos \\
Desarrollo socioemocional & $\rightarrow$ Seguridad y protección \\
& $\rightarrow$ Autorregulación emocional \\
& $\rightarrow$ Afectividad \\
\hline
\end{tabular}

\subsection{Técnicas e instrumentos de investigación}

En primer lugar, se aplicó la técnica de observación no participante mediante el empleo de un cuaderno de campo como instrumento de recolección de la información relativa a las tres categorías de estudio predeterminadas. Esta labor estuvo a cargo de una de las investigadoras, quien efectuó un total de diez observaciones de clase en un periodo de tres meses, durante los cuales asistió una vez a la semana a la institución en el horario de 8 a.m. a 12 m.

Al finalizar las observaciones, se aplicó, como técnica de investigación, una entrevista a la docente con preguntas referidas a cada categoría abordada en el estudio, considerando como instrumento una guía de entrevista semiestructurada, basada en cinco preguntas disparadoras, orientadas a identificar los modos de interacción con sus estudiantes y el tipo de disciplina que, según la docente, ejerce en el aula, así como las características socioemocionales y cognitivas de sus estudiantes. Cabe señalar que esta guía fue validada mediante la técnica de juicio de expertos en la que se contó con la participación de tres especialistas en el tema, quienes brindaron su apreciación y retroalimentación frente a cada ítem. La evaluación dio como resultado una fiabilidad fuerte (se alcanzaron los 3 puntos según la escala de Likert, lo cual representó el puntaje máximo).

\subsection{Procedimiento de recolección y procesamiento de datos}

Para la recolección de los datos se solicitó el permiso de la directora de la Institución quien aceptó la ejecución del estudio en el centro educativo. Además, ella se encargó de comunicar a los padres de familia acerca de la presencia continua de la investigadora. Por otra parte, se coordinó con la docente el día de visita semanal al colegio, así como la fecha de la entrevista. 
En la primera sesión, la profesora presentó a la investigadora, de tal modo que los alumnos supieran que ella los acompañaría en el lapso de tres meses y que observaría su comportamiento en clase.

Luego de haber recopilado los datos, se procedió a ejecutar el procesamiento de información, para lo cual se empleó principalmente el Atlas ti, software que facilitó la integración y contraste de los datos cualitativos. Además, para el análisis e interpretación, se utilizó la triangulación de resultados mediante el empleo de matrices de organización con el fin de establecer aquellos aspectos comunes, producto de la confluencia de los dos instrumentos de investigación aplicados.

\section{Resultados}

El análisis de los resultados se llevó a cabo mediante los datos obtenidos a través de la observación realizada durante tres meses de clase, así como de la entrevista aplicada a la docente. Es preciso señalar que el análisis se efectuó teniendo en cuenta las categorías apriorísticas consideradas para la investigación. Cabe aclarar que, para resguardar la privacidad de los participantes, en la declaración de los hallazgos, no se han incluidos los nombres verdaderos de los estudiantes ni de la docente, a quien se suele aludir como «la docente», «la maestra» $\mathrm{o}$ «la profesora».

\subsection{Estilo de disciplina e interacción docente- estudiantes}

A partir de las observaciones realizadas se evidenció que, al inicio de cada jornada, la docente recibía a sus estudiantes con gestos molestos o serios sin ninguna muestra de afecto. Los estudiantes, por su parte, tampoco demostraban cercanía hacia su profesora. Durante las sesiones, se observó un trato vertical entre la docente y los estudiantes, ya que, constantemente, a través de gritos, llamadas de atención violentas, condicionamientos radicales o situaciones de subordinación, ejercía poder y excesiva autoridad sobre ellos, lo cual los llevaba a sentir incomodidad extrema o temor, como varios de ellos manifestaban entre sí. Algunas expresiones empleadas por los estudiantes fueron las siguientes: "Cállate, que va a seguir gritando", "Ya no hay que decir nada», "Me duelen los oídos», entre otras.

Ejemplos de conductas agresivas por parte de la docente son los golpes que ella propinaba en la mesa o en la pared del aula para llamar la atención de los estudiantes, así como los gritos compulsivos dirigidos a aquellos estudiantes que, en clase, discutían entre sí. Un aspecto que llamó la atención fue el tipo de comunicación ejercido con los estudiantes. Al respecto, se observó que, ante situaciones de desobediencia de los niños como salir del aula sin permiso 
para ir al baño, ella les solía recriminar con enunciados expresados en tono humillante como: "¿a quién le has pedido permiso para ir al baño?, "¿así de malcriado eres en tu casa?» Otra expresión utilizada por la docente fue la siguiente: "Por desobediente te sacas malas notas». Asimismo, se evidenció que la docente solía llamar la atención con mayor ímpetu a aquellos estudiantes que, según su percepción, siempre se comportaban mal. Por ejemplo, ante un caso de dos niños que discutían porque querían utilizar el mismo lápiz de color para pintar, la docente optó por golpear la mesa y gritar a uno de ellos: "iRoberto, toda la vida tú! Estamos mejor los días que no vienes; ahi todos estamos tranquilos y felices». Enseguida, sin preguntar qué era lo que estaba ocurriendo, lo mandó directamente a la oficina del promotor. En una ocasión, se observó, además, que, ante el caso de una alumna que sentía dolores en el estómago, la docente le recriminó echando la culpa a sus padres: "Tu mamá se pasa; ¿cómo te va a mandar así, mal del estómago?». Luego de ello, optó por llamar a los padres para que la recogieran. Ante tal situación, no se observó mayor acercamiento por parte de la docente para calmar a la alumna. Un ejemplo de condicionamiento que solía ejercer la docente para mantener la disciplina en clase era la costumbre de permitir salir al recreo solo a aquellos alumnos que se comportaban correctamente en clase.

En la Figura 1, se puede observar el porcentaje de ocurrencia de conductas violentas mostradas por la docente, teniendo en cuenta las doce sesiones de clase observadas.

Figura 1. Conductas violentadas mostradas por la docente.

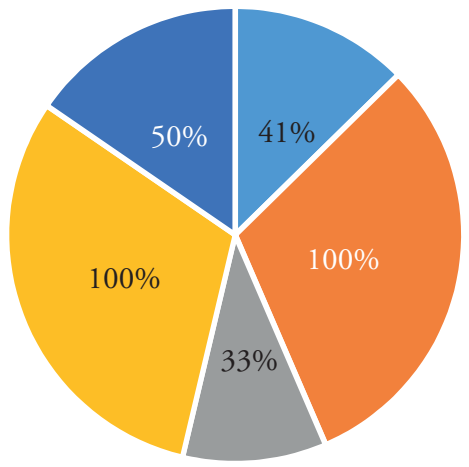

Golpes en la mesa o pared

Llamadas de atención violentas a todo el grupo

Expresiones humillantes o inadecuadas hacia los estudiantes

Gritos dirigidos a estudiantes desobedientes

Condicionamientos radicales

Asimismo, durante las horas de clase, mientras los niños resolvían sus fichas de trabajo, la docente permanecía con su celular y actuaba de manera indiferente ante las demandas de los niños, lo cual los llevaba a la frustración y desesperación por no saber cómo resolver las actividades. Esto se manifestó 
a partir de los gestos de inquietud emitidos por los estudiantes, así como las quejas entre ellos al manifestar su poca comprensión ante las consignas brindadas por la profesora. Algunas expresiones utilizadas por los alumnos fueron las siguientes: "¿qué se hace?", "no entiendo", "y ahora, ¿qué hacemos?».

En relación con el trato individualizado que la profesora brindaba a sus estudiantes, se evidenció principalmente agresividad e indiferencia. Se observó un trato cordial solamente con uno de los estudiantes, lo cual se reflejó con un tono de voz amable o concesivo; justamente, este estudiante era el más tranquilo de la clase. En la Figura 2, se muestran los porcentajes del tipo de trato otorgado por la docente a cada uno de sus estudiantes.

Figura 2. Trato individualizado docente- estudiantes.

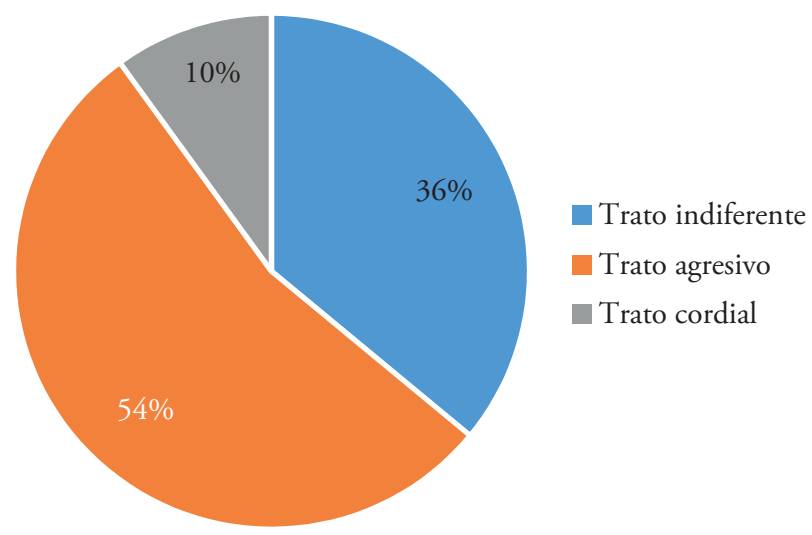

Los hallazgos anteriores se oponen a la información brindada en la entrevista por la docente, quien resaltó la necesidad de impartir la disciplina en el aula "de manera consensuada», mediante el siguiente tipo de acciones:

Es importante hablar con los niños. Por ejemplo, supongamos, hoy todos los niños que trabajan bien en el aula van a tener un premio por portarse bien, $O$ puede ser que les diga que saldremos al parque para jugar con los juegos. O también se les pone caritas felices y un premio, ya sea un caramelo, ya que de esa manera a los niños se les hace más felices.

Asimismo, según la docente, la disciplina debe impartirse «explicando al alumno que lo que va a hacer en un momento dado puede generar consecuencias negativas». Al respecto, la profesora manifestó la necesidad de hacer respetar las normas de convivencia. No obstante, ello no se evidenció en ninguna de las sesiones observadas. 


\subsection{Desarrollo cognitivo}

Es preciso afirmar que la maestra del aula no atendía a los intereses de los niños, dado que solo atinaba a hacerlos completar fichas de trabajo. De este modo, de las doce observaciones realizadas, solo en dos sesiones se evidenció el uso de material gráfico que permitiera a los estudiantes desarrollar su estimulación sensorial.

Por otro lado, sus sesiones no solían contar con los momentos pedagógicos correspondientes, por lo que no las iniciaba con una motivación que despertara expectativas y emociones en los nińos. Ello se opone con lo señalado por la docente en la entrevista realizada, pues, desde su percepción, ella despierta la motivación de sus estudiantes «mediante videos, o sea con materiales audiovisuales». Además, indicó que "es bueno, para lograr un mejor aprendizaje, el uso del juego", lo cual tampoco se demostró en las clases observadas.

Asimismo, se evidenció una casi nula exploración física y social por parte de los estudiantes; ya que la docente prefería la aplicación de tareas individuales en vez de grupales, pese a que estas últimas son de mayor interés para el alumno. Esto se confirmó en una de las sesiones observadas, en la cual la docente hizo que los alumnos realizaran una actividad gráfico-plástica en grupo. En ella los estudiantes participaron de muy buen ánimo en los equipos formados y trabajaron grupalmente sin ningún problema.

En relación con el desarrollo de procesos cognitivos, tales como la percepción, la memoria, la atención y la metacognición, no se evidenció, en las sesiones de clase, la realización de actividades orientadas a ejercitar tales operaciones. De las doce sesiones observadas, solo en tres de ellas la profesora hizo un recordatorio sobre los temas trabajados en la clase anterior para vincularlos con el tema de la sesión. Más bien, se evidenció que la docente, a lo largo de las sesiones, necesitaba recordar continuamente a los alumnos los temas que estaban trabajando.

En la entrevista realizada a la docente, manifestó un bajo desempeño académico de, al menos, el $50 \%$ de sus estudiantes. Ello concuerda con las observaciones de clase realizadas, en donde se comprobó que la mayoría de los estudiantes mostraban dificultades para resolver los ejercicios de clase y preferían desarrollar alguna otra actividad en vez de preguntar a la docente. Ahora bien, la profesora atribuye este bajo desempeño a diversas causas como "aspectos psicológicos, falta de interés de los padres, problemas familiares». Así, desde la percepción de la docente, el aburrimiento y el desorden generados en el aula se deben a los problemas que los estudiantes "traen de casa». Pese a ello, durante las sesiones observadas, no se evidenció un trabajo personalizado por parte de la profesora para apoyar a los estudiantes que tenían esas características. 


\subsection{Desarrollo socioemocional}

En las sesiones de clase se observaron pocas interacciones positivas entre la profesora y sus estudiantes, así como un nulo acercamiento de los alumnos hacia ella. Como ya se mencionó anteriormente, los niños manifestaban verbalmente su frustración ante la incomprensión de las actividades de clase que debían desarrollar. Igualmente, se evidenció por parte de la docente una actitud desinteresada ante las necesidades afectivas de sus alumnos, al preferir el empleo del celular mientras los mantenía ocupados realizando alguna actividad de clase pese a que pocos la completaban, mientras que los demás optaban por caminar en el aula, hablar con sus compañeros o salir del salón. Tal situación se vincula con el componente afectivo que forma parte del desarrollo socioemocional. Asimismo, como ya se indicó anteriormente, se observaron situaciones de subordinación excesiva que la docente ejercía sobre los estudiantes, lo cual no permitía el desarrollo de vínculos afectivos positivos entre ambos actores educativos.

Con relación a la autorregulación emocional, no se desarrollaron habilidades de este tipo en los estudiantes. En las sesiones observadas, con pocas excepciones, primaba el desorden y el descontrol. Ello se vio reflejado a través de peleas entre los niños, situaciones constantes de distracción, y al llanto de algunos estudiantes que se sentían incómodos o frustrados ante las reacciones agresivas de la docente. Igualmente, no se observó, en ninguna de las clases, la generación de espacios de diálogo con sus estudiantes en los que ellos pudieran expresar sus sentimientos para aprender a autorregularse.

Los hallazgos anteriores son opuestos a la postura manifestada por la maestra en la entrevista. La docente considera que es «importante que un niño sea feliz, ya que eso ayuda a que un niño no tenga problemas psicológicos y pueda aprender mejor. Si un niño es feliz quiere decir que los padres son felices». Pese a ello, no se evidenció, en ninguna de las sesiones observadas, acciones destinadas a lograr la estabilidad emocional de los alumnos y un clima de clase positivo. Por otro lado, la docente manifestó que, para la solución de conflictos, considera que «todos los alumnos deben compartir los diferentes materiales, juegos e incluso no pelearse entre ellos. También pedir disculpas cuando es necesario, ya que a ellos no les gustaría pasar por malos tratos». Las respuestas brindadas son contradictorias al tipo de prácticas disciplinarias ejercidas en el aula, pues no se constató que ella incentive en sus estudiantes el desarrollo de estas actitudes socioemocionales. 


\section{DisCUSIÓN Y CONCLUSIONES}

A partir de los hallazgos, es preciso afirmar que el estilo de disciplina impartido por la docente corresponde a la disciplina obediente (Minedu, 2010, citado en Córdova, 2013). Dicho estilo hace referencia a un trato vertical entre el docente y el alumno haciendo uso de normas coactivas, lo cual permite afirmar que la docente aplica un estilo autoritario y, a su vez, negligente, según la clasificación de Ellis y Hughes (2002). De esta forma, como señalan Zovko y Dilon (2018), no se muestra respeto a las necesidades, intereses y demandas del alumnado. Incluso, pese a que la docente es consciente de los problemas familiares de algunos alumnos, lo que perjudica su rendimiento académico, no realiza acciones orientadas a atenderlos de manera personalizada, pese a que este tipo de enseńanza constituye supuestamente uno de los principios rectores de la institución. Más bien, se observó una saturación de actividades poco productivas para los estudiantes.

Ahora bien, el estilo de la docente se caracterizó por la aplicación de prácticas sumamente perjudiciales para el desarrollo de los infantes, tales como golpes en objetos, gritos desmedidos, frases humillantes, condicionamientos extremos e, incluso, castigos sobre la base de prejuicios y no de hechos comprobados. Al respecto, cabe señalar que los castigos que la docente aplica como por ejemplo, evitar que los alumnos desobedientes salgan al patio a la hora de recreo, son inadecuados, pues, según Soto (2017), el castigo debe ser la última opción, luego de intentar aplicar otro tipo de procedimientos correctivos. Tal situación es preocupante, pues como indican Bwire et al. (2020), este estilo de disciplina perjudica el desarrollo académico y socioafectivo de los menores. Por el contrario, se ha comprobado que un estilo de disciplina consciente o positivo genera muchos más beneficios, además de incrementar los niveles de motivación (Aragáo et al., 2017; Chávez et al., 2017; Jiménez, 2018; Silupu, 2019).

En relación con el desarrollo cognitivo de los estudiantes, la docente no aplica estrategias pedagógicas para lograr un aprendizaje significativo y que este corresponda con las necesidades, intereses y desarrollo evolutivo del niño, pese a que se debe construir las estructuras cognoscitivas a partir de prácticas continuas con el medio (Piaget, s/f, citado en Crain y Dunn, 2007). No existe un trabajo pedagógico sostenido orientado a la estimulación sensorial ni a la exploración física y social. Las actividades que plantea son, principalmente, mecánicas y no siempre pueden ser resueltas por el alumnado. Cabe precisar, además, que el clima de clase es inadecuado, caracterizado por el mal trato de la docente, así como por situaciones de indisciplina que no permiten el 
fortalecimiento de las operaciones cognitivas de los alumnos como la atención, el razonamiento, la memoria y la metacognición (Best, 2002 ). Esto se evidenció en las actitudes demostradas por los alumnos, quienes tenían muchas dificultades en concentrarse en sus materiales de clase y en recordar las indicaciones de la docente, quien debía repetirles varias veces el tema de la sesión. A ello se suma la carencia de actividades de recojo de saberes previos por parte de la profesora, lo cual podría ayudar a los estudiantes en la realización de mejores conexiones neuronales (Redolar, 2014).

Además de todo lo anterior, resalta la inexistencia del juego como recurso de enseñanza, lo que permitiría afianzar muchos de los procesos cognitivos que los alumnos requieren adquirir (Peralta, 2013). Igualmente, se evidencia la realización de pocas actividades que consigan involucrar, de manera motivada, a los estudiantes. Tal problemática se produce, pese al desempeño académico bajo que evidencian los estudiantes, como la misma docente afirmó en la entrevista, lo cual se corrobora en las observaciones de clase. Así, la baja motivación de los alumnos, además de sus evidentes problemas de indisciplina, no les permiten superar sus falencias de nivel cognitivo, y, más bien, estas se agravan, pues la docente continúa avanzando los temas de clase sin reparar en el refuerzo que los estudiantes requieren para adquirir los aprendizajes deseados.

En cuanto al desarrollo socioemocional de los alumnos, se observaron situaciones de peleas entre ellos y de frustración con llantos como medio para exteriorizar sus emociones negativas, todo lo cual podría generar estrés en el alumnado y, así, alterar significativamente sus procesos cognitivos (Campos, 2010). Por otro lado, tales actitudes están directamente vinculadas con su falta de habilidades socioemocionales, las cuales son necesarias para establecer interacciones eficaces con sus pares y con sus cuidadores (Sánchez, 2008). En tal sentido, al no haber un vínculo positivo entre docente y estudiantes, la formación socioemocional resulta perjudicada (Molina y Pérez, 2006). Por otro lado, no se evidenciaron estrategias orientadas a desarrollar la competencia socioemocional de los estudiantes, lo cual resulta negativo, pues ello permitiría una mejor convivencia entre ellos, a la vez que posibilitaría la formación de habilidades de autorregulación de sus emociones (Garaigordobil, 2018).

A partir de la integración de los hallazgos, es preciso afirmar que el tipo de disciplina ejercida por la docente, al igual que sus prácticas y actitudes negativas perjudican el desarrollo emocional y cognitivo de sus estudiantes. En primer lugar, los gritos, rechazos y amenazas impartidos por la maestra para controlar la desorganización y distracción de sus alumnos generan algún impacto emocional en el alumnado, reflejado en sentimientos de frustración 
y desmotivación en su proceso de aprendizaje. En segundo lugar, el hecho de que los niños estén inmersos en un contexto en el cual el trato es violento podría generar la normalización de prácticas agresivas como medio de resolución de conflictos, tal como lo plantean Juárez y Márquez (2010). Ello interviene negativamente en su formación socioemocional. En tercer lugar, se evidencian situaciones que podrían catalogarse como maltrato infantil con el pretexto de la disciplina (Mazadiego, 2005), lo que indiscutiblemente podría estar afectando el desarrollo cognitivo y socioemocional del alumnado: en adición, los educadores deben constituir un ejemplo de inteligencia emocional para los niños. De ahí parte la necesidad de velar y regular la actitud afectiva que rodea su comunicación con los infantes (Cabello, 2011). No obstante, ello no ocurre en el caso observado ya que la docente, más bien, demuestra una falta de inteligencia emocional, aunado al deficiente manejo de estrategias para mejorar la disciplina de los estudiantes (Quispe, 2018).

A modo de conclusión, es importante tener en cuenta que los estímulos emocionales interactúan con las habilidades cognitivas. Si bien es cierto, a pesar de que, en la entrevista realizada a la docente, ella haya asegurado de que motiva a sus alumnos de diversas maneras, tal afirmación no es más que una falacia en contraste con lo observado. Por el contrario, se evidenció un trato negligente y excesivamente autoritario por parte de la maestra, lo cual podría estar resultando sumamente dañino para los estudiantes, tanto a nivel cognitivo como socioemocional.

Para finalizar, entre las limitaciones del presente estudio, resalta la imposibilidad de recolectar información directa de los propios estudiantes, debido a las directivas institucionales. Asimismo, al ser un estudio de caso, los hallazgos no pueden ser generalizados. No obstante, sí pueden aportar en el diseńo de futuros estudios que se realicen sobre este tema, sobre todo, considerando el contexto de educación infantil y los factores relacionados con la aplicación de estilos de disciplina violenta para, así, posibilitar el diseño de programas de formación docente orientados a desterrar estilos de disciplina violenta.

\section{REFERENCIAS BIBLIOGRÁFICAS}

Albornoz, E. J. y Guzmán, M. C. (2016). Desarrollo cognitivo mediante estimulación en niños de 3 años. Universidad y Sociedad, 8(4), 186-192. Recuperado de http://rus.ucf.edu.cu/

Aragão, M., Dos Santos, M. N. y De Andrade, A. (2017). Disciplina positiva: possibilidades para repensar os castigos escolares no contexto da educaçao infantil. Encontro Internacional de Formação de Professores e Fórum 
Permanente de Inovação Educacional, 10(1). Recuperado de https://eventos. set.edu.br/enfope/article/view/4860/1495

Best, J. (2002). Psicología cognoscitiva. México D.F.: Thomson Learning.

Bisquerra, R. y Pérez, N. (2007). Las competencias emocionales. Educación XXI(10), 61-82. https://doi.org/10.5944/educxx1.1.10.297

Bwire, F., Nkuba, M., Hermenau, K. y Hecker, T. (2020). Reducing violent discipline by teachers using Interaction Competencies with Children for Teachers (ICC-T): study protocol for a matched cluster randomized controlled trial in Tanzanian public primary schools. Trials, 21(4), 1-11. https://doi.org/10.1186/s13063-019-3828-z

Cabello, M. T. (2011). Importancia de la inteligencia emocional como contribución al desarrollo integral de los niño/as de educación infantil. Pedagogía Magna, (11), 178-188. Recuperado de https://dialnet.unirioja.es/descarga/ articulo/3629180.pdf

Campos, A. L. (2010). Neuroeducación: Uniendo las neurociencias y la educación en la búsqueda del desarrollo humano.LaEduc@ción. Recuperado de http:// kdoce.cl/wp-content/uploads/2017/10/DOC1-neuroeducacion.pdf

Chávez, M. C., Ramos, A. y Velázquez, P. Z. (2017). Análisis de las estrategias docentes para promover la convivencia y disciplina en el nivel de educación preescolar. Educación, 26(51), 55-78. https://doi.org/10.18800/ educacion.201702.003

Crain, G. y Dunn, W. (2007). Understanding human development. New Jersey: Pearson Prentice-Hall.

Córdova, B. G. (2013). La disciplina escolar y su relación con el aprendizaje en el área de Historia, Geografía y Economía de los alumnos del 4 to año secciones " $A$ " $Y$ " $B$ " de educación secundaria de la institución educativa "San Miguel» de Piura. (Tesis de maestría). Universidad de Piura. Piura, Perú. Recuperado de https:// pirhua.udep.edu.pe/bitstream/handle/11042/1819/MAE_EDUC_113.pdf

Ellis, D. y Hughes, K. (2002). Connecting schools, families, and communities for youth success. Cultivating effectives and meaningful School-Family-Community Partnerships. Portland: Northwest Regional Educational Laboratory.

Espot, M. R. (2011). La autoridad del profesor: Qué es la autoridad y cómo se adquiere. Barcelona: Wolters Kluwer Educación.

Garaigordobil, M. (2018). La educación emocional en la infancia y la adolescencia. Participación Educativa, 5(8), 105-128.

Guedeney, A. y Dupong, I. (2020). The effects of socio-affective environment. Handbook of Clinical Neurology, 173, 443-450. https://doi.org/10.1016/ B978-0-444-64150-2.00031-9 
Jiménez, M. E. (2018). Disciplina positiva y la modulación del comportamiento de estudiantes de educación general básica en el Ecuador. (Tesis de maestría). Universidad Andina Simón Bolívar. Quito, Ecuador. Recuperado de http://repositorio.uasb.edu.ec/bitstream/10644/6139/1/T2642-MIEJimenez-Disciplina.pdf

Juárez, J. y Márquez, A. (2010) El maltrato infantil en la práctica docente: Estudio de un caso. Psicogente, 12(21), 158-181. Recuperado de https://www. redalyc.org/pdf/4975/497552353012.pdf

Lafrancesco, V. G. M. (2003). La educación integral en el preescolar: Propuesta pedagógica. Bogotá: Magisterio.

Larrañaga, A. (2012). El modelo educativo tradicional frente a las nuevas estrategias de aprendizaje. (Trabajo fin de máster). Universidad Internacional de la Rioja, Bilbao. Recuperado de https://reunir.unir.net/bitstream/handle/ 123456789/614/Larra\%C3\%B1aga\%20Ane.pdf?sequence=1

Llorent, V., Zych, I. y Varo-Millán, J.C. (2020). Competencias socioemocionales autopercibidas en el profesorado universitario en España. Educación, 23(1), 297-318. https://doi.org/10.5944/educxx1.23687

Márquez, G. J. y Díaz, N. J. y Cazzato, D. S. (2007). La disciplina escolar: aportes de las teorías psicológicas. Revista de Artes y Humanidades UNICA, 8(18). Recuperado de https://www.redalyc.org/pdf/1701/170118447007.pdf

Mazadiego, M. T. (2005). Detección de maltrato infantil en una muestra de escuelas primarias. Enseñanza e Investigación en Psicología, 10(2), 281-293. Recuperado de https://www.redalyc.org/pdf/292/29210205.pdf

Molina, N. y Pérez, I. (2006). El clima de relaciones interpersonales en el aula: un caso de estudio. Paradigma, 27(2), 193-219.

Nelsen, J. (2015). Disciplina positiva. São Paulo: Manole.

Peralta, A. (2013). Importancia de la educación socioemocional en el jardín de infantes. Buenos Aires: Dunken.

Pérez, N. y Filella, G. (2019). Educación emocional para el desarrollo de competencias emocionales en niños y adolescentes. Praxis \& Saber, 10(24), 23-44. https://doi.org/10.19053/22160159.v10.n25.2019.8941

Pulido, F. y Herrera, F. (2017). La influencia de las emociones sobre el rendimiento académico. Ciencias Psicológicas, 11(1), 29-39. https://doi.org/10.22235/ cp.v11i2.1344

Quispe, J. (2018). Manejo de estrategias docentes para mejorar la disciplina de los estudiantes de la IEP No 70174 de Pomata. (Trabajo de Segunda Especialidad). Universidad San Ignacio de Loyola, Lima, Perú. Recuperado de http://200.37.102.150/bitstream/USIL/6511/3/2018_QUISPE_SUCASACA_JUSTO_PASTOR.pdf 
Redolar, D. (2014). Neurociencia cognitiva. Madrid: Editorial Médica Panamericana.

Sánchez, R. (2008). El desarrollo emocional de los niños en edad preescolar. (Trabajo de grado de licenciatura). Universidad Pedagógica Nacional, México D.F. Recuperado de http://200.23.113.51/pdf/25989 .pdf

Santrock, J. W. (2004). Desarrollo infantil. Madrid: Mc Graw Hill.

Silupu, E. (2019). Manifestaciones de disciplina positiva en preescolares de 5 años, en Carabayllo, Lima 2019. (Trabajo de grado de licenciatura). Universidad César Vallejo, Lima, Perú. Recuperado de https://repositorio.ucv.edu.pe/bitstream/ handle/20.500.12692/43533/Silupu_ZEC.pdf?sequence=1\&isAllowed $=\mathrm{y}$

Soto, B. (2017). Modificación de la conducta agresiva en niños, postulados de Skinner según la teoría de condicionamiento operante referente al castigo. (Trabajo de grado de licenciatura). Universidad Técnica de Machala, Machala, Ecuador. Recuperado de http://repositorio.utmachala.edu.ec/bitstream/48000/ 10548/1/ECUACS\%20DE00023.pdf

Zamudio, V. R. (2010). Disciplina escolar: Desarrollo y aplicación de un programa actitudinal-cognitivo para la formación permanente del profesorado de educación primaria. (Tesis de doctorado). Universitat Autónoma de Barcelona. Barcelona, España. Recuperado de https://www.tdx.cat/bitstream/handle/ 10803/32144/rzv1de1.pdf?sequence $=1$ \&isAllowed $=y$

Zovko, M.E. y Dillon, J. (2018). Humanism vs. competency: Traditional and contemporary models of education, Educational Philosophy and Theory, 50(6), 554-564. https://doi.org/10.1080/00131857.2017.1375757 